\title{
Constructs of Sequence Pairs with Optimal Three-level Autocorrelation
}

\author{
Jin Hui-long ${ }^{1}$, Zhang Miao ${ }^{2}$ and Qi Shu-li ${ }^{2}$ \\ ${ }^{1}$ Hebei Normal University, The Faculty of Electronic, Shijiazhuang 050024, China \\ ${ }^{2}$ Xidian University, Communication Engineering, Xi'an 710071, China \\ ${ }^{1} 13131145063 @ 163 . c o m$
}

\begin{abstract}
The optimal discrete signal are widely used in spread spectrum communication systems, sonar, navigation, code division multiple access systems and other fields. The perfect sequence pair is an extension of the single best signal, so its application is more widely. This paper puts forward new methods of constructing the binary sequence pair of three values and quaternion sequence pair of three values according to the problem of constructing the sequence pairs of three values. This method is that it uses $N$ long cyclotomic pseudo-random sequence and its shift sequences pair to do series connection to constitute a $4 \mathrm{~N}$ long binary sequence pair of three values. New sequence pairs of three values is obtained by this method proved to have good balance. The application steps of this method are given and shall be verified by some examples.
\end{abstract}

Keywords: Optimum signal, Sequence pair of three values, Cyclotomic class, Autocorrelation

\section{Introduction}

It is an important indicator to evaluating whether the pseudo random sequence is of good autocorrelation and balance characteristic during designing pseudo random sequence signal in the radar, sonar code division multiple access systems [1]. If the autocorrelation of a sequence will satisfy the following two conditions it says that this sequence has the best correlation: 1) Values of the sequence of non-synchronous autocorrelation are as little as possible; 2) The number of sequence of non-synchronous autocorrelation values is as less as possible [2]. If the length of sequence is even and this sequence is of the same number of "1" and "0", it argues that this sequence is balance, while if the difference number of "1" and" 0 " is only 1 , it argues that this sequence is almost balanced; If the sequence length is odd and the difference number of " 1 " and " 0 " is only 1 , it argues that this sequence is balanced [3].

So far, for the construction of the binary sequence, there have been some very good results, but not perfect. T. Storer, C. Ding and others proved that the composite construction which is equivalent to the binary sequence having optimal relevance is a difference set or almost difference sets with specific parameter [4-5]. As a result, the optimal or the optimal relevance in the series of the relatedness of the construction of the binary sequence, the combination design method and algebraic method occupies an extremely important position, the use of limited domain points round division and round number division is the important method to construct the binary sequence [6-7].

By difference set and almost difference set, people have constructed many balanced an $d$ almost balanced optimal autocorrelation sequence of three values [8-10], and such seque nces have good linear complexity [11]. For autocorrelation sequence of three values of per $\operatorname{iod} v$, if $v \equiv 0(\bmod 4)$, then $R(\tau) \in\{0,-4\}$ or $R(\tau) \in\{0, \pm 4\}$; if $v \equiv 1(\bmod 4)$, then $R(\tau) \in\{1,-3\}$; if $v \equiv 2(\bmod 4)$, then $R(\tau) \in\{2,-2\}$; if $v \equiv 3(\bmod 4)$, then $R(\tau)=-1$, where $R(\tau) \quad(0<\tau<v-1$ 
) is non-synchronous autocorrelation values of sequence [12].

To enlarge the existence of sequence space, people have discussed from many aspects, including that it puts forward the concept of "pairs" in literature [13] which is with sequence pairs forming with two sequences to form an optimal signal. When the sequence is applied to a system in the original project, in the system the sender and the receiver must use the same sequence. Now as long as the sequence pairs forming with the sending sequence and local sequence used in the receiver can satisfy certain conditions, such a sequence coupling is formed a best signal. Based on the above principle, the literature [14, 15] proposed the sequence pairs of three values having good balance characteristics. This method greatly relaxed the selection range of sequence and more conducive to looking for the best signal.

\section{The Basic Concepts}

Definition 2.1 [16]: For binary $(1,+1)$ sequence pair $(s, t)$ of the period $N$, if the sequence pair $(s, t)$ in the autocorrelation function $R_{(s, t)}(\tau)$ can be expressed as

$$
R_{(s, t)}(\tau)= \begin{cases}F \neq 0, \pm 1, & \tau=0 \\ 1, & \tau \neq 0\end{cases}
$$

Then this sequence pair $(s, t)$ is called fake random binary sequence pairs.

Definition 2.2: Let $\mathrm{S}(\mathrm{n})$ be a binary periodic sequence, cycle for $\mathrm{N}$, if set A meets the following conditions

$$
A=\{0 \leq n \leq N-1: S(n)=1\}
$$

Then set $A$ is called as supported sequence of sequence $S(n)$. And the $S(n)$ is called as the characteristics sequence of set $\mathrm{A}$, where $A \subseteq Z_{N}=\{0,1,2, \ldots, N\}$.

Define 2.3: Let $v=e f+1$ be exponential, $F_{v}$ for $v$ order finite field, $F_{v}{ }^{*}=F_{v} \backslash\{0\}$ and let $w$ as the origin of $F_{v}, \varepsilon=\omega^{e}, H_{i}^{e}=\left\{w^{i}, w^{i} \varepsilon, w^{i} \varepsilon^{2}, \cdots, w^{i} \varepsilon^{f-1}=w^{i}<\varepsilon>\right\}$, $0 \leq i \leq e-1$

Where $H_{0}^{e}, H_{1}^{e}, \cdots, H_{e-1}^{e}$ is called the $e$ order of round division class of $F_{q}$. When it needs not to indicate $e$, it is also shorthand $H_{i}^{e}$ for always $H_{i}$.

Definition 2.4 [17]: Let $D, D^{\prime}$ be the two subsets of v order Abel group F, $k, k^{\prime}$ be the number of elements in the collection $D, D^{\prime}$, i.e. $k=|D|, k^{\prime}=|D|$, e is the number of the same element in $D, D^{\prime}$, i.e. $e=\left|D \mathrm{I} D^{\prime}\right|$, if any nonzero element in the difference table $\left\{d-d^{\prime} \mid d \neq d^{\prime}, d \in D, d^{\prime} \in D^{\prime}\right\} \quad$ in $\mathrm{F}$ happens to appear $\lambda$ times, then $\left(D, D^{\prime}\right)$ is said as one of difference set pairs $\left(v, k, k^{\prime}, e, \lambda\right)-$ on $\mathrm{F}$.

Theorem 2.1 [18]: Let the prime power $v=2 f+1$, when $v \equiv 3(\bmod 4)$ and $f$ is an odd number, then $\left(H_{0} \cup\{0\}, H_{1}\right)$ constitutes a set of differential pair, and the difference pairs $(s, t)$ corresponding to this sequence pairs is called round division pseudo random sequence pair.

Example 2.1 When $v=11$, it is easy to know that $H_{0}=(0,1,3,4,5,9),\left(H_{0} \cup\{0\}, H_{1}\right)$, $H_{1}=(2,6,7,8,10)$ constitutes a $(11,6,5,3)$ set of differential - pairs. And it can construct a round division pseudo random sequence pair, where $s=(--+---+++)$, 
$t=(++-+++---+-)$ and the autocorrelation function is

$$
R_{(s, t)}(\tau)=\sum_{i=0}^{10} s_{i} t_{i+\tau}=\left\{\begin{array}{ll}
-11 & \tau=0 \\
1 & \tau \neq 0
\end{array} .\right.
$$

Note: For prime power $v=2 f+1$ and $f$ is odd and is odd prime number within 100 . Within 100, when $v$ only takes $3,7,11,19,23,31,47,59,67,71,79,83$, it can get the corresponding round division pseudo random sequence pair by theorem 2.1 .

\section{The Construction Method of the Binary Sequence Pairs of Three Values}

Equipped with sequence set $\left\{a_{0}, a_{1}, \ldots, a_{T-1}\right\}$ and $\left\{b_{0}, b_{1}, \ldots, b_{T-1}\right\}$ where $a_{i}(i=0,1, \ldots, T-1)$ and $b_{i}(i=0,1, \ldots, T-1)$ are the best binary sequences with period $N$.If concentrated continuous sequences are connected, they form a sequence $u$ and $v$ of period $N T$, remember $u=I\left(a_{0}, a_{1}, \ldots, a_{T-1}\right)$, $v=I\left(b_{0}, b_{1}, \ldots, b_{T-1}\right)$. Where $I$ denotes connection operator here.

If the cyclic shift left of the sequence $v$ is to remember $L^{\tau}(v)$, where $\tau=T \tau_{1}+\tau_{2}$ $\left(0 \leq \tau_{1}<N, 0 \leq \tau_{2}<T\right)$, so they have

$$
L^{\tau}(v)=I\left(L^{\tau_{1}}\left(b_{\tau_{2}}\right), \ldots, L^{\tau_{1}}\left(b_{T-2}\right), L^{\tau_{1}+1}\left(b_{0}\right), \ldots, L^{\tau_{1}+1}\left(b_{\tau_{2}-1}\right)\right) .
$$

So the cross-correlation function at the shift of $\tau$ of the two connected sequences $u$ and $v$ is just the sum of inner product of the sequence of columns of the sequence $u$ and $v$.As

$$
R_{(u, v)}(\tau)=\sum_{i=0}^{T-\tau_{2}-1} R_{a_{i}, b_{i+\tau_{2}}}\left(\tau_{1}\right)+\sum_{i=T-\tau_{2}}^{T-1} R_{a_{i}, b_{i+\tau_{2}-T}}\left(\tau_{1}+1\right)
$$

Theorem 3.1: Let $(a, b)$ and $(s, t)$ be two round division pseudo random binary sequence pairs with the period $N$, where $N \equiv 3(\bmod 4)$. Then it tells us that this two round division pseudo random binary sequence can construct binary sequence pairs $(u, v)$ of three values of period $4 N$,

where $u=I\left(a, L^{\frac{N+1}{4}}(s), L^{\frac{N+1}{2}}(a), L^{\frac{3(N+1)}{4}}(s)+1\right), v=I\left(b, L^{\frac{N+1}{4}}(t), L^{\frac{N+1}{2}}(b), L^{\frac{3(N+1)}{4}}(t)+1\right)$

The autocorrelation function of them is

$$
R_{(u, v)}(\tau)=\left\{\begin{array}{l}
-4 N, 1(\text { time }) \\
0,3 N(\text { times }) 0 \leq \tau<4 N \text { Equation (4-11) } \\
4, \quad N-1 \text { (times ) }
\end{array}\right.
$$

Proof: Remember

$\tau=T \tau_{1}+\tau_{2},\left(0 \leq \tau_{1}<N\right.$ and $\left.0 \leq \tau_{2}<4\right)$, so the autocorrelation function values can be to consider from several things $\tau_{2}=0,1,2,3$. 
(1) When $\tau_{2}=0$, there is $0<\tau_{1}<N$. According to the (4-11), the sequence $v$ at

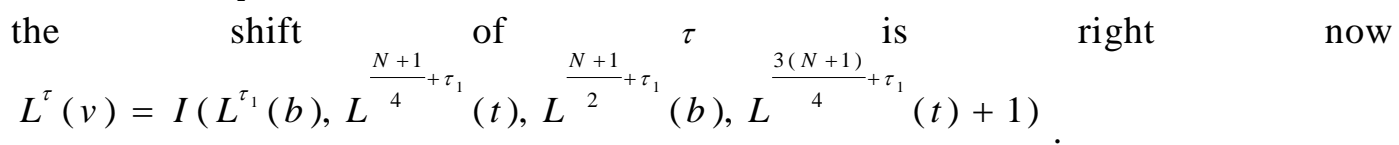

So the autocorrelation function value is

$$
\begin{aligned}
R_{(u, v)}(\tau)= & R_{(a, b)}\left(\tau_{1}\right)+R_{(s, t)}\left(\tau_{1}\right)+R_{(a, b)}\left(\tau_{1}\right)+R_{(s, t)}\left(\tau_{1}\right) \\
& =4
\end{aligned}
$$

In addition, when $\tau_{2}=0$ and $\tau_{1}=0$, there is

$$
R_{(u, v)}(\tau)=R_{(a, b)}(0)+R_{(s, t)}(0)+R_{(a, b)}(0)+R_{(s, t)}(0)
$$

And because sequence pairs $(a, b),(s, t)$ are all round division pseudo random binary sequence pairs with cycle $N \equiv 3(\bmod 4)$. So all its autocorrelation function value is $-N$ at the point $\tau=0$.

$$
\begin{aligned}
\therefore R_{(u, v)}(\tau)= & R_{(a, b)}(0)+R_{(s, t)}(0)+R_{(a, b)}(0)+R_{(s, t)}(0) \\
& =-4 N .
\end{aligned}
$$

(2) When $\tau_{2}=1$ there is $T=4$ according to the (4-11). At the same time the sequence $v$ at the shift of $\tau$ is right now

$$
L^{\tau}(v)=I\left(L^{\tau_{1}+\frac{N+1}{4}}(t), L^{\frac{N+1}{2}+\tau_{1}}(b), L^{\frac{3(N+1)}{4}+\tau_{1}}(t)+1, L^{\tau_{1}+1}(b)\right)
$$

So the autocorrelation function value is

$$
\begin{aligned}
& R_{(u, v)}(\tau)=R_{(a, t)}\left(\tau_{1}+\frac{N+1}{4}\right)+R_{(s, b)}\left(\tau_{1}+\frac{N+1}{2}-\frac{N-1}{4}\right) \\
& \quad-R_{(a, t)}\left(\tau_{1}+\frac{3(N+1)}{4}-\frac{N+1}{2}\right)-R_{(s, b)}\left(\tau_{1}+N+1-\frac{3(N-1)}{4}\right) \\
& =R_{(a, t)}\left(\tau_{1}+\frac{N+1}{4}\right)+R_{(s, b)}\left(\tau_{1}+\frac{N+1}{4}\right)-R_{(a, t)}\left(\tau_{1}+\frac{N+1}{4}\right)-R_{(s, b)}\left(\tau_{1}+\frac{N+1}{4}\right) \\
& =0
\end{aligned}
$$

(3)When $\tau_{2}=2$ there is $T=4$ according to the (4-11). At the same time the se quence $v$ at the shift of $\tau$ is right now

$$
L^{\tau}(v)=I\left(L^{\tau_{1}+\frac{N+1}{2}}(b), L^{\frac{3(N+1)}{4}+\tau_{1}}(t)+1, L^{\tau_{1}+1}(b), L^{\tau_{1}+1+\frac{N+1}{4}}(t)\right),
$$

So the autocorrelation function value is

$$
\begin{aligned}
& R_{(u, v)}(\tau)=R_{(a, b)}\left(\tau_{1}+\frac{N+1}{2}\right)-R_{(s, t)}\left(\tau_{1}+\frac{3(N+1)}{4}-\frac{N-1}{4}\right) \\
+ & R_{(a, b)}\left(\tau_{1}+N+1-\frac{N+1}{2}\right)-R_{(s, t)}\left(\tau_{1}+N+1+\frac{N+1}{4}-\frac{3(N-1)}{4}\right) \\
= & 2 R_{(a, b)}\left(\tau_{1}+\frac{N+1}{2}\right)-2 R_{(s, t)}\left(\tau_{1}+\frac{N+1}{2}\right) \\
= & 0 .
\end{aligned}
$$


Because $(a, b)$ and $(s, t)$ are round division pseudo random sequence pairs that the values of $R_{(a, b)}(\tau)$ and $R_{(s, t)}(\tau)$ are equal when the delay time $\tau$ is not equal to zero.

(3) When $\tau_{2}=3$ there is $T=4$ according to the (4-11). At the same time the sequence $v$ at the shift of $\tau$ is right now

$$
L^{\tau}(v)=I\left(L^{\tau_{1}+\frac{3(N+1)}{4}}(t)+1, L^{\tau_{1}+1}(b), L^{\frac{N+1}{4}+\tau_{1}+1}(t), L^{\tau_{1}+1+\frac{N+1}{2}}(b)\right),
$$

So the autocorrelation function value is

$$
\begin{aligned}
& R_{(u, v)}(\tau)=-R_{(a, t)}\left(\tau_{1}+\frac{3(N+1)}{4}\right)+R_{(s, b)}\left(\tau_{1}+N+1-\frac{N-1}{4}\right) \\
& +R_{(a, t)}\left(\tau_{1}+N+1+\frac{N+1}{4}-\frac{N+1}{2}\right)-R_{(s, b)}\left(\tau_{1}+N+1+\frac{N+1}{2}-\frac{3(N-1)}{4}\right) \\
& =R_{(a, t)}\left(\tau_{1}+\frac{3(N+1)}{4}\right)+R_{(s, b)}\left(\tau_{1}+\frac{3(N+1)}{4}\right) \\
& -R_{(a, t)}\left(\tau_{1}+\frac{3(N+1)}{4}\right)-R_{(s, b)}\left(\tau_{1}+\frac{3(N+1)}{4}\right)=0 .
\end{aligned}
$$

Finish.

Remark: In two pairs of cyclotomic pseudo-random sequence pair $(a, b),(s, t)$ for constructing three value binary sequence pair $(u, v)$ with period $4 N,(s, t)$ can be the shift, complementary transformations of $(a, b)$, or unequivalent with $(a, b)$.

Therom 3.2: Three value binary sequence pair $(u, v)$ is balanced, that is $N_{0}(u, v)=N_{1}(u, v)=4 N$.

Proof: In cyclotomic pseudo-random sequence pairs $(a, b),(s, t)$, with period $N \equiv 3(\bmod 4)$, the elements in sequence $b$ are complementary to the corresponding ones of $a$, and the elements in sequence $s$ are complementary to the corresponding ones of $t$, too.

$$
u=I\left(a, L^{\frac{N+1}{4}}(s), L^{\frac{N+1}{2}}(a), L^{\frac{3(N+1)}{4}}(s)+1\right) v=I\left(b, L^{\frac{N+1}{4}}(t), L^{\frac{N+1}{2}}(b), L^{\frac{3(N+1)}{4}}(t)+1\right)
$$

So in three values binary sequence pair $(u, v), N_{0}(u, v)=N_{1}(u, v)=4 N \quad(u, v)$ is balanced.

Example 3.1: Let $(a, b),(s, t)$ be two cyclotomic pseudo-random sequence pairs with period 7 , so the constructing method of three value binary sequence pair $(u, v)$ with period 28 is as following:

First, let $(a, b)=(1,0,0,1,0,1,1,0,1,1,0,1,0,0)$ be a cyclotomic pseudo-random sequence pair, $(s, t)$ is the let shift transformation of $(a, b)$, shift value $\Gamma=1$, so $(s, t)=(0,0,1,01,1,1,1,1,0,1,0,0,0)$ is a cyclotomic psudo-random sequence pair.

Result:

$$
\begin{gathered}
u=I\left(a, L^{\frac{N+1}{4}}(s), L^{\frac{N+1}{2}}(a), L^{\frac{3(N+1)}{4}}(s)+1\right)= \\
(1,0,0,1,0,1,1,1,0,1,1,1,0,0,0,1,1,1,0,0,1,0,1,1,0,1,0,0), \\
v=I\left(b, L^{\frac{N+1}{4}}(t), L^{\frac{N+1}{2}}(b), L^{\frac{3(N+1)}{4}}(t)+1\right)=
\end{gathered}
$$


$(0,1,1,0,1,0,0,0,1,0,0,0,1,1,1,0,0,0,1,1,0,1,0,0,1,0,1,1)$.

Therefore, calculate the autocorrelation function values description of $(u, v)$ is $\left(R_{(u, v)}(\tau)\right)_{\tau=0}^{27}=(-28,0,0,0,4,0,0,0,4,0,0,0,4,0,0,0,4,0,0,0,4,0,0,0,4,0,0,0)$.

\section{Analyzing the Performance to Resist the Noise}

In this paper, the perfect punctured sequence pairs of the length 61 i.e.

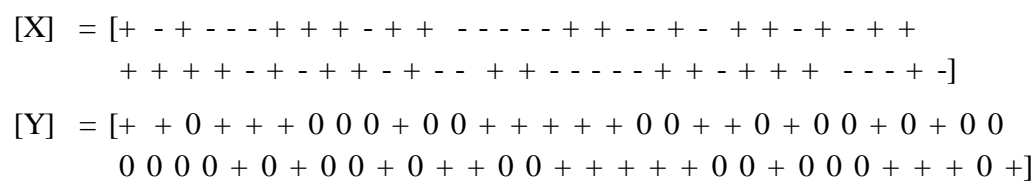

is applied to series of MSK modulation in direct sequence spread spectrum system(SMSK - DSSS). And then it does comparison analysis via simulation to m sequence and Gold se quence with the length of 63 and the 45th line sequence of 64 order Hadamard matrix.

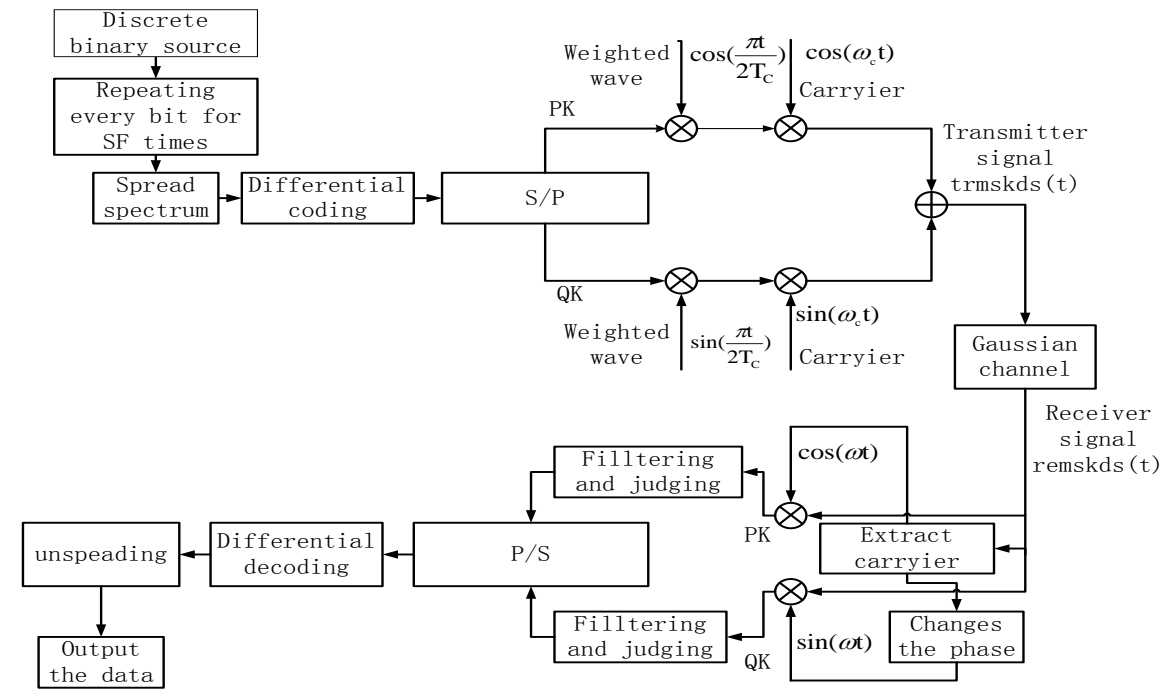

Figure 1. The Simulation Principle Block Diagram of SMSK-DSSS

PART ONE. The Simulation Principle Block Diagram of SMSK - DSSS

According to the literature, we get to know that the orthogonal expression of MSK signal is as follows:

$$
\begin{gathered}
\left.\left.\operatorname{SMSK}(\mathrm{t})=\mathrm{cos} \omega_{\mathrm{c}}(\mathrm{t}) \cos (\mathrm{t})\left(2 \mathrm{~T}_{\mathrm{f}}\right)\right) \operatorname{co} \varphi_{\mathrm{k}}-\mathrm{A}_{\mathrm{k}} \sin \omega_{\mathrm{c}}(\mathrm{t}) \sin (\mathrm{t})(2 \mathrm{~T})\right) \operatorname{si} \varphi_{k} \\
=\mathrm{P}_{\mathrm{k}} \cos \omega_{\mathrm{c}}(\mathrm{t}) \cos (\pi \mathrm{t}) /\left(2 \mathrm{~T}_{\mathrm{b}}\right)-\mathrm{Q}_{\mathrm{k}} \sin \omega_{\mathrm{c}}(\mathrm{t}) \sin (\pi \mathrm{t}) /\left(2 \mathrm{~T}_{\mathrm{b}}\right)
\end{gathered}
$$

Where $P_{k}$ is called synthetic component, while $Q_{k}$ is called orthogonal component. And there are:

$$
\begin{aligned}
& \mathrm{P}_{\mathrm{k}}=\cos \mathrm{os}_{\mathrm{k}} \\
& \mathrm{Q}_{\mathrm{k}}=\operatorname{sinp_{k}} ; \varphi_{\mathrm{k}}=0 \text { or } \pi .
\end{aligned}
$$


By the expression, it is easy to build the simulation principle block diagram which is shown in Figure 1 of SMSK-DSSS. And in the process of simulation we assume that both the arrier and PN sequences keep strict synchronization.

PART TWO. Perfect Punctured Sequence Pairs of Antinoise Performance Comparison Analysis.

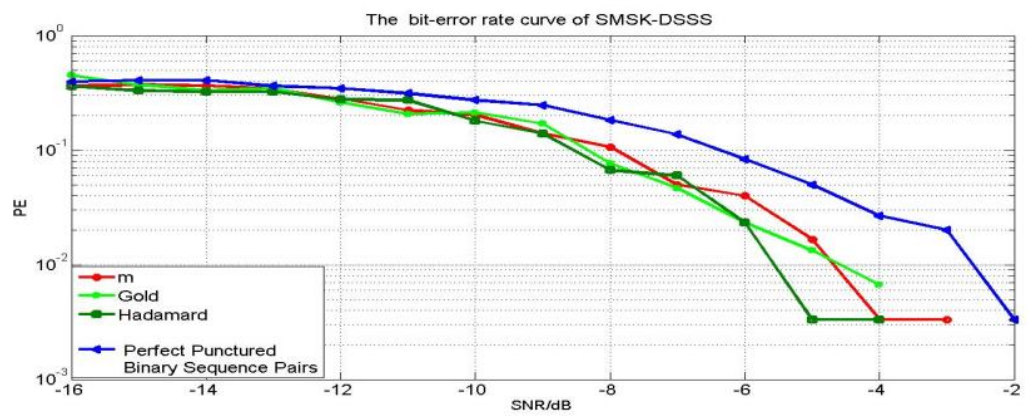

Figure 2. The Bit-error Rate Curve of SMSK-DSSS when Applying m, Gold, Hadamard Respectively and Perfect Punctured Sequence Pairs

As shown in Figure 2. the aforementioned perfect punctured sequence pairs of the length 61 is used to SMSK-DSSS, after comparing with Gold sequence, m sequence and Hadamard we get the bit-error rate curve of SMSK-DSSS. From the figure we can analyze that the system bit error rate curve is close when applying m, Gold and Hadamard since the signal-to-noise ratio SNR changes from -16 to -2 . By the figure, when using the $\mathrm{m}$ sequence and Hadamard the system bit error-rate curve is volatile while using Gold sequence relatively stable. But it can be seen that when applying the perfect punctured sequence pairs the performance of resisting noise of the system is worst. In order to more intuitive, we list the error-rate data tables below whose values vary from -10 to -4 .

Table 4.3.1. The Comparison Table of the System Bit-error Rate when Applying $\mathrm{m}$, Gold, Hadamard and the Perfect Punctured Sequence Pairs

\begin{tabular}{|c|c|c|c|c|c|c|c|}
\hline & $10^{-}$ & 9 & 8 & 7 & 6 & 5 & 4 \\
\hline $\mathrm{m}$ & 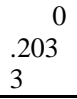 & $\begin{array}{l}0 \\
.140 \\
0 \\
\end{array}$ & $\begin{array}{l}{ }^{1} \\
.106 \\
7 \\
\end{array}$ & $\begin{array}{l}\quad 0 \\
.050 \\
0\end{array}$ & $\begin{array}{l}\quad 0 \\
.040 \\
0\end{array}$ & $\begin{array}{lr} & 0 \\
.016 & \\
7 & \end{array}$ & $\begin{array}{l}{ }^{1}{ }^{0} \\
.003 \\
3\end{array}$ \\
\hline d $\quad$ Gol & $\begin{array}{l}{ }^{0} \\
.213 \\
3\end{array}$ & $\begin{array}{l}0^{0} \\
.170 \\
0\end{array}$ & $\begin{array}{l}\quad 0 \\
.076 \\
7\end{array}$ & $\begin{array}{l}0^{0} \\
.046^{2} \\
7\end{array}$ & $\begin{array}{l}0^{0} \\
.023 \\
3^{2}\end{array}$ & $\begin{array}{l}{ } \begin{array}{l}0 \\
.013 \\
3\end{array} \\
\end{array}$ & $\begin{array}{l}\quad 0 \\
.006 \\
7\end{array}$ \\
\hline $\mathrm{H}$ & $\begin{array}{l}{ }^{0} \\
.180 \\
0 \\
0\end{array}$ & $\begin{array}{l}0 \\
.140 \\
0 \\
0\end{array}$ & $\begin{array}{l}\quad 0 \\
.066 \\
7 \\
\end{array}$ & $\begin{array}{l}\quad 0 \\
.060 \\
0 \\
\end{array}$ & $\begin{array}{l}{ }^{1}{ }^{0} \\
.023 \\
3^{2} \\
\end{array}$ & $\begin{array}{l}{ }^{1} 0 \\
.003 \\
3 \\
\end{array}$ & $\begin{array}{l}{ }^{1} 0 \\
.003 \\
3 \\
\end{array}$ \\
\hline $\mathrm{P}$ & $\begin{array}{l}{ }^{0} \\
.273 \\
3\end{array}$ & $\begin{array}{l}0 \\
.246 \\
7\end{array}$ & $\begin{array}{l}{ }^{1}{ }^{0} \\
.183 \\
3^{2}\end{array}$ & $\begin{array}{l}{ }^{1} 0 \\
.136 \\
7\end{array}$ & $\begin{array}{l}{ }^{1}{ }^{0} \\
.083 \\
3^{2}\end{array}$ & $\begin{array}{l}{ }^{1}{ }^{0} \\
.050 \\
0\end{array}$ & $\begin{array}{l}{ }^{1} \\
.026 \\
7\end{array}$ \\
\hline
\end{tabular}

Note: $\mathrm{H}$ represents the Hadamard; P represents the perfect punctured sequence

\section{Conclusion}

In this paper, the cyclotomic psudo-random sequence pair get from reference, bring forward two construction methods of three value sequence pairs. Firstly, cyclotomic psudo-random sequence pairs are known in this method, then shift these sequence pairs, according to the shift numbers different sequence pairs can be achieved. Usually, choose a known sequence pair and some kind of its shift transformations, then connect the sequences of sequence pairs and do Gray reflection, as a result, new kind of balanced 
three value binary sequence pairs or three value quarternary sequence pair can be achieved. Through the study in this paper, the theory of sequence pair is enriched as well as brings forward more discrete signals with better characteristics in engineering applications.

\section{Acknowledgment}

This work is supported by National Natural Science Foundation of China (NSFC), China (No61172094), by Natural Science Foundation of Hebei Province, China.( No. F2011205103)

\section{References}

[1] Y. Yixian, “Theory and Design of Perfect Signal”, the People's Posts \& Telecom Press, (1996), Beijing, pp. 56-78.

[2] X. Tang and C. Ding, "New classes of balanced quaternary and almost balanced binary sequences with optimal autocorrelation value", IEEE Trans. Information Theory, vol. 56, vol. 12, (2010), pp. 63986405.

[3] C. Ding and X. Tang, "The cross correlation of binary sequences with optimal autocorrelation", IEEE Trans. Information Theory, vol. 56, no. 4, (2010), pp. 1694-1701.

[4] T. Storer, "Cyclotomy and difference sets, Markham publish company", Chicago, (1967), pp. 25- 83.

[5] C. Ding, T. Helleseth and K. Y. Lam, "Several classes of sequences with three-level auto-correlation", IEEE Tran. Inform. Theory, vol. 47, no. 11, (1999), pp. 2606-2612.

[6] M. Yukiyasu and D. Vladimir, "Difference systems of sets and cyclotomy", Discrete Mathe-matics, vol. 308, no. 7, (2008), pp. 2959-2969.

[7] X. H. Tang and G. Gong, "New constructions of binary sequences with optimal autocorrelation value/magnitude", IEEE Trans. Inf. Theory, vol. 56, no. 3, (2010), pp. 1278-1286.

[8] C. Ding, T. Helleseth and H. M. Martinsen, "New families of binary sequences with optimal three-level autocorrelation", IEEE Tran. Inform. Theory, vol. 47, no. 1, (2001), pp. 428- 433.

[9] G. J. Ness and T. Helleseth, "A New Three-Valued Cross Correlation Between-Sequences of Different Lengths”, IEEE. Trans. Inf. Theory, vol. 52, no. 10, (2006), pp. 4695-4701.

[10] T. Helleseth, A. Kholosha and G. J. Ness, "Characterization of Sequences of Lengths and With Three-Valued Cross Correlation", IEEE Trans. Information Theory, vol. 53, no. 6, (2007), pp. 2236-2245.

[11] Q. Wang and X. Du, "The linear complexity of binary sequences with three-level autocorrelation", IEEE Trans. Information Theory, vol. 56, no. 8, (2010), pp. 4046- 4052.

[12] C. Ding and X. Tang, "The Cross-Correlation of Binary Sequences With Optimal Autocorrelation", IEEE Trans. Information Theory, vol. 56, no. 4, (2010), pp. 1694-1701.

[13] Z. Xiao-qun, H. Wen-cai and W. Zhong-wen, "The theory of the perfect binary array pairs", Electronica Sinica, vol. 27, no. 1, (1999), pp. 34-37.

[14] X. Peng, C. Xu and K. T. Arasu, "New Families of Binary Sequence Pairs With Two-Level and Three-Level Correlation", IEEE Trans. Information Theory, vol. 58, no. 11, (2012), pp. 6968-6978.

[15] J. Huilong, C. Jiaxing and X. Chengqian, "Study on Construction of a Class Three-level of Autocorrelation Sequence Pair”, Journal of Convergence Information Technology, vol. 8, no. 1, (2013), pp. 475-480.

[16] L. Zhaobin, J. Ting and Z. Wei-xia, "Reasearch on a new spead sequence pairs", Dianzi Yu Xinxi Xuebao, vol. 31, no. 4, (2009), pp. 889-892.

[17] X. Cheng-qian, "Differences set pairs and approach for the studly of perfet binary array pairs",. Acta Electronica Sinica, vol. 29, no. 1, (2001), pp. 87-89.

[18] J. Hui-long and X. Cheng-qian, "The Study of Methods for Constructing a Family of Pseudorandom Binary Sequence Pairs Based on the Cyclotomic Class", Acta Electronica Sinica, vol. 38, no. 7, (2010), pp. 1608-1611.

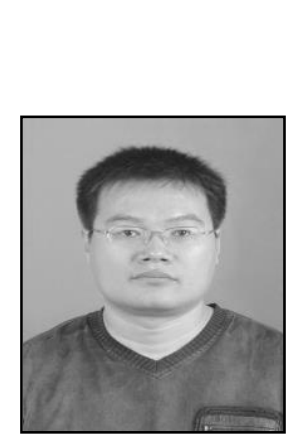

\section{Author}

Jin Hui-long, born in 1973, Ning Jin County. He Bei province, Doctor in Circuit and System Profession, YanShan University, in 2012. Now work in Hebei normal university as an adjunct professor, mainly study Coding Theory, Cryptogrm, Perfect Discrete Signal Desin. 